\title{
Generation of induced pluripotent stem cells using skin fibroblasts from patients with myocardial infarction under feeder-free conditions
}

\author{
JUN-QUAN LI $^{1 *}$, MING CHENG $^{1 *}$, WEI-CHEN TIAN ${ }^{1}$ and JIAN ZHANG ${ }^{2}$ \\ ${ }^{1}$ Department of Cardiac Surgery, The Second Affiliated Hospital, Harbin Medical University, Nangang, Harbin, \\ Heilongjiang 150086; ${ }^{2}$ Department of Surgery, The Affiliated Hospital of Medical College, \\ Qingdao University, Qingdao, Shandong 266003, P.R. China \\ Received June 25, 2013; Accepted December 18, 2013
}

DOI: $10.3892 / \mathrm{mmr} .2014 .1885$

\begin{abstract}
Myocardial infarction (MI) is an increasing medical problem; however, its pathogenesis has yet to be elucidated and more effective treatment strategies are required. Induced pluripotent stem cells (iPSCs) were recently successfully generated using human somatic cells transfected with four transcription factors. The present study aimed to generate iPSCs from cells from patients with myocardial infarction. Six patients who had been diagnosed with myocardial infarction were enrolled in this study. The fibroblast cells from the biopsied skin were reprogrammed using octamer-binding transcription factor 4 (Oct-4), SRY-related HMG-box gene 2 (Sox-2), Kruppel-like factor 4 (Klf-4) and cellular myelocytomatosis oncogene (c-Myc) transcription factors. The generated cells were identified by karyotyping, in vitro and in vivo differentiation ability and staining for specific markers. These human MI-iPSCs expressed pluripotent genes and cell surface markers, and exhibited normal proliferation. The iPSCs also showed in vivo and in vitro differentiation ability, as indicated by teratoma and embryoid body formation, respectively. Moreover, the iPSCs differentiated into cardiomyocytes and neuronal cells. In conclusion, human iPSCs were successfully generated from skin fibroblasts from patients with MI under feeder-independent conditions, which increases their
\end{abstract}

Correspondence to: Dr Jian Zhang, Department of Surgery, The Affiliated Hospital of Medical College, Qingdao University, 16 Jiangsu Road, Qingdao, Shandong 266003, P.R. China

E-mail: zhangjianmedmail@126.com

Dr Wei-Chen Tian, Department of Cardiac Surgery, The Second Affiliated Hospital, Harbin Medical University, 246 Xuefu Road, Nangang, Harbin, Heilongjiang 150086, P.R. China

E-mail: tianweichendoctor@gmail.com

*Contributed equally

Key words: induced pluripotent stem cells, myocardial infarction, reprogramming potential suitability for clinical applications. These results may encourage further study of MI pathogenesis and facilitate the development of safe downstream clinical applications of iPSC-based cell therapies.

\section{Introduction}

Induced pluripotent stem cells (iPSCs) show tremendous potential for application in regenerative medicine, and have been successfully derived from mice (1), rats (2), monkeys (3) and humans (4) since 2006. Moreover, iPSCs derived from patients provide opportunities to study the pathogenesis of various diseases. To date, somatic cells from patients with type I diabetes (5), kidney disease (6), Lesch-Nyhan syndrome (7), Gaucher's disease (7), muscular dystrophy (7) and neurodegenerative disorders (7) have been successfully reprogrammed into human iPSCs.

The prevalence of cardiomyopathy is increasing worldwide, and it has been the leading cause of death in the United States for the past 80 years. Human iPSCs were recently generated from patients with Down syndrome, but few studies have derived iPSCs from cardiac patients $(8,9)$. Myocardial infarction (MI) results from interruption of the blood supply to part of the heart, causing the death of cardiac cells. There is currently no effective treatment for MI (10); however, cell therapy using stem cells may represent a potential therapeutic strategy. Certain studies have indicated that myocardial cells can be derived from human embryonic stem cells (ESCs) $(11,12)$ and iPSCs $(13,14)$; however, it is necessary to generate the iPSCs from the specific patient in order to study the pathogenesis of the disease and investigate potential gene modifications for treating genetic heart disease.

Murine fibroblast cells from 12.5- or 13.5-day-old fetuses are traditionally used as feeder cells to support the growth of iPSCs and ESCs $(4,15)$. However, animal-derived feeder cells are associated with the risk of transmitting animal pathogens to human ESCs, and are therefore not desirable in cell lines intended for transplantation in humans (16). Only specific types of fibroblast cells are generally employed to support human pluripotent stem cell growth, including foreskin and abdominal fibroblasts $(17,18)$. This indicates that pluripotent 
stem cells can be derived from human fibroblast feeder cells, thus avoiding the risk of animal contamination. Moreover, the effects of heterologous feeder cells on human iPSCs remain to be elucidated, even when both cell types originate from humans. The development of feeder-free conditions is therefore key to the further application of iPSCs.

Feeder-free conditions have been applied in the derivation and propagation of human ESCs $(19,20)$, as well as human iPSCs (21); however, the use of feeder-free conditions to generate human iPSCs from cardiac patients has yet to be reported. Therefore, the present study generated human MI-iPSCs using feeder-free conditions and mouse fibroblast feeder layers, and compared the characteristics of the iPSCs derived from the two systems in terms of cell surface markers and in vitro and in vivo differentiation capacities.

\section{Materials and methods}

All reagents were purchased from Sigma-Aldrich (St. Louis, MO, USA), unless otherwise indicated.

Skin biopsy and preparation of a human fibroblast cell line. The present study was approved by the Henan Provincial People's Hospital (Zhengzhou, Henan, China), Tianjin Central Hospital of Obstetrics and Gynecology (Tianjin, China), and Affiliated Hospital of Medical College, Qingdao University (Qingdao, Shandong, China), and no financial benefits were involved in the donation process. Written informed consent was obtained from the patients. It was guaranteed to the donors that biopsied tissue and the resulting fibroblast cells were only for use in basic scientific research. One piece of skin tissue was obtained by biopsy from each of six patients with heart disease. The tissue was minced with scissors following rinsing in phosphate-buffered saline (PBS), and dissociated into single cells using $0.5 \%$ trypsin. The cells were then transferred to culture medium containing $85 \%$ Dulbecco's Modified Eagle's medium supplemented with $15 \%$ fetal bovine serum, 2 mM L-glutamine, $1 \%$ minimum essential medium nonessential amino acids and $1 \%$ penicillin-streptomycin. These primary-passage fibroblast cells were divided following seven days of incubation, prior to being passaged every three to four days. Cells at passage four were prepared for iPSC generation.

Generation of iPSCs by lentivirus transduction. A lentivirus with octamer-binding transcription factor 4 (Oct-4), SRY-related HMG-box gene 2 (Sox-2), Kruppel-like factor 4 (Klf-4) and cellular myelocytomatosis oncogene (c-Myc) was provided as a commercial kit (SCR544; Millipore, Billerica, MA, USA), and the procedure for generating iPSCs was performed according to the product specifications. Fibroblast cells were prepared and used as they reached $30 \%$ confluence. On the first day, $1 \mu$ l lentivirus solution $\left(10^{9} \mathrm{TU} / \mathrm{ml}\right)$ was added to the cell culture medium together with $8 \mu \mathrm{g} / \mathrm{ml}$ polybrene. The culture medium was changed on the second day, and a further $1 \mu \mathrm{l}$ lentivirus solution was added with $8 \mu \mathrm{g} / \mathrm{ml}$ polybrene. The culture medium with lentivirus was discarded on the third day and new culture medium was added. The fibroblast culture medium was changed to a stem cell culture medium on the sixth day, and the cells were digested with $0.25 \%$ trypsin. The iPS cell culture medium consisted of
80\% KO-DMEM, 20\% KO-serum, 1\% L-glutamine, $0.5 \%$ penicillin/streptomycin, $1 \%$ non-essential amino acids, $1 \mathrm{mM}$ $\beta$-mercaptoethanol and $10 \mu \mathrm{g} / \mathrm{ml}$ bFGF The transfected cells were then plated onto mitomycin $\mathrm{C}$-treated mouse embryo fibroblast cells, and cultured for 12 days until small colonies appeared. After 20 days, the colonies were removed using a glass needle and cut into pieces. One part was cultured in Matrigel-coated tissue culture dishes, and the other part was cultured in mouse feeder cell-coated tissue culture dishes. The iPSCs were passaged every four to seven days.

Karyotyping analysis of iPSCs. Karyotyping analysis was performed as previously described (15). iPSCs were treated with colchicine and blocked at mitotic metaphase. The nuclear membrane was then broken by potassium chloride treatment. Following fixation in methanol:glacial acetic acid, the cells were transferred onto glass slides, the chromosome spreads were stained with Giemsa and their images were captured. The chromosomes were visualized using standard G-band staining. For each sample, $\geq 50$ metaphase cells were examined.

Immunofluorescence analysis of iPSCs. Stem cells were characterized using specific stem cell markers. iPSCs were fixed using $4 \%$ paraformaldehyde following rinsing with PBS, and subsequently incubated with the primary antibodies to tumor rejection antigen-1-60 (TRA-1-60; MAB4381, Millipore), Oct-4 (ab27985) and Sox2 (ab75485, Abcam, Cambridge, UK) at $1: 100$ dilution overnight at $4^{\circ} \mathrm{C}$. The cells were then washed with PBS and the secondary antibody (1:200) was added immediately. The secondary antibody was fluorescein isothiocyanate (FITC)-conjugated antibody (ab97224) and Texas Red-conjugated antibody (ab6757) (Abcam). Cell nuclei were stained with Hoechst 33342 (Sigma-Aldrich). Cells were examined using a confocal laser scanning system (LSM 510 META; Carl Zeiss, Jena, Germany).

Gene expression analysis by reverse transcription-polymerase chain reaction ( $R T-P C R)$. Total RNA was extracted using a TRIzol ${ }^{\circledR}$ RNA isolation kit (Invitrogen Life Technologies, Carlsbad, CA, USA) according to the manufacturer's instructions. Cytoplasmic RNA from human iPSCs and their derived embryoid bodies (EB) were reverse-transcribed to single-stranded cDNA. This cDNA was subjected to PCR amplification using primers for genes representative of the three germ layers, including $\alpha$-fetoprotein (AFP, endoderm), NEUOD-1 (ectoderm) and HBZ (mesoderm).

Differentiation ability in vitro. The differentiation ability of iPSCs was analyzed in vitro by EB formation, as previously described (15). Clumps of iPSCs were suspended in culture medium without basic fibroblast growth factor, following digestion with $1 \mathrm{mg} / \mathrm{ml}$ type IV collagenase. EB formation was observed five to seven days later, and the three germ layers were analyzed regarding their representative gene expression.

Differentiation ability in vivo. iPSC colonies were digested using $1 \mathrm{mg} / \mathrm{ml}$ type IV collagenase, and $2-5 \times 10^{5}$ cells were collected and injected into the back leg of severe combined immunodeficiency (SCID) mice. The SCID mice were purchased from the animal center in the Second Affiliated 
A

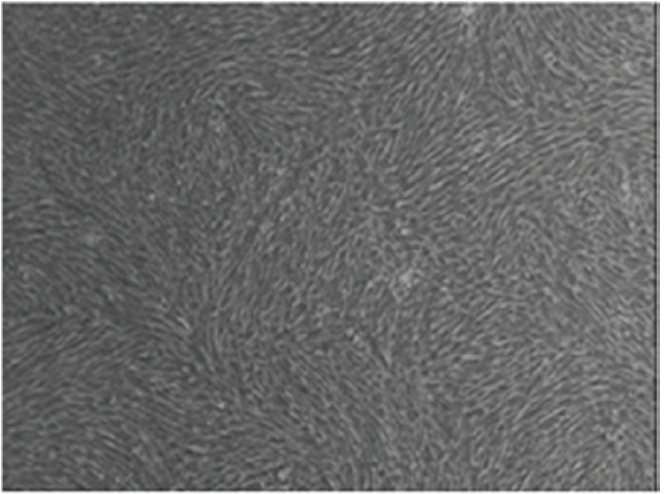

B

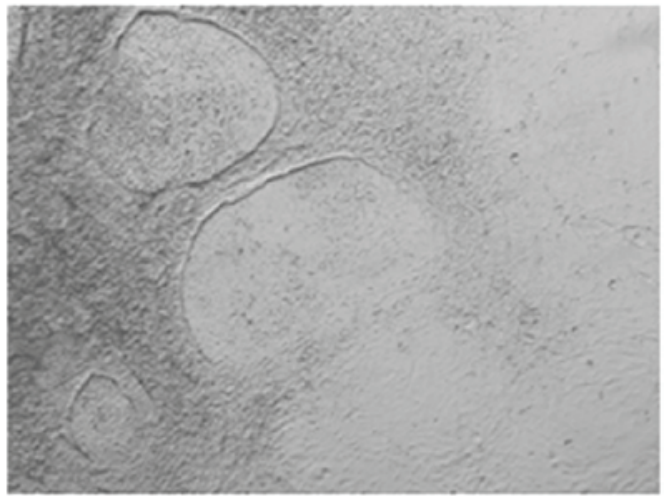

C

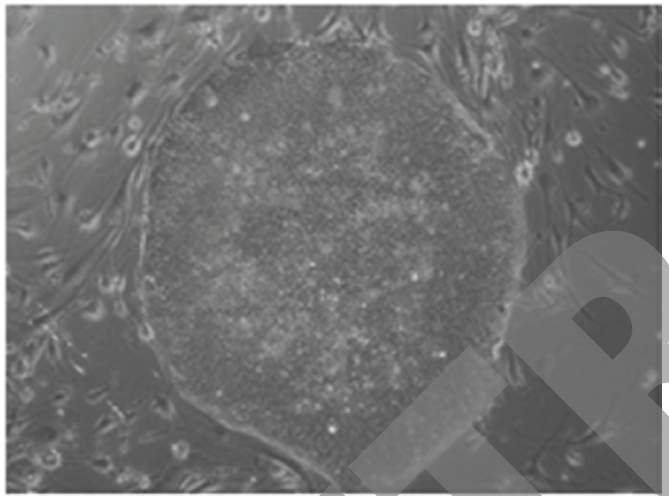

D

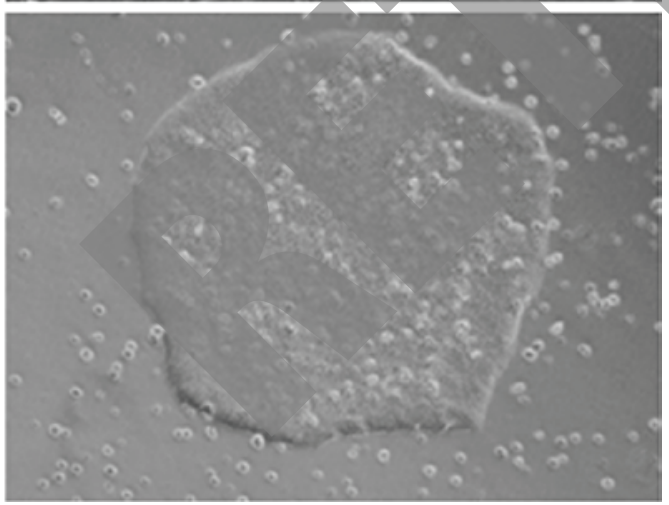

Figure 1. Morphology of somatic cells from patients with MI and the resulting human iPS cell colonies. (A) Fibroblast cells from patients with MI at passage 4. Magnification, $x 40$; (B) primary colonies at day 20 after transfection with the four transcription factors. Magnification, x100; (C) human iPS cell colony on the feeder layers. Magnification, x100; (D) human iPS cell colony under feeder-free conditions. Magnification, x100. iPS, induced pluripotent stem cells; MI, myocardial infarction.

Hospital, Harbin Medical University. The production and sale licenses were obtained from the ZPSTD, Inspection and Quarantine Bureau (Harbin, China) and Price Bureau (Harbin,
A

B

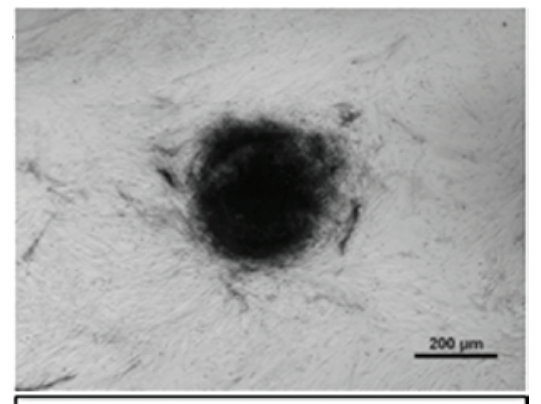

C
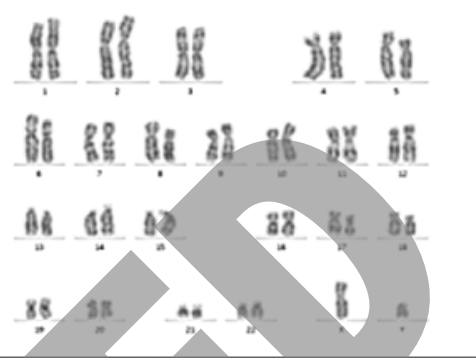

if 1 i

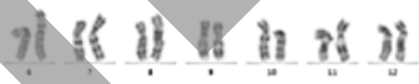

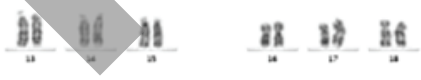

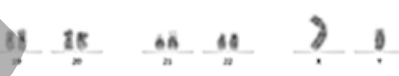

Figure 2. AP staining and karyotyping analysis of human iPS cells (A) AP staining of a human iPS cell colony; (B) karyotyping of human iPS cells at passage 15; (C) karyotyping of human iPS cells at passage 30. iPS, induced pluripotent stem cells; AP, alkaline phosphatase.

China). These mice were fed in accordance with national legislation for animal care. Teratomas, which developed after three months, were biopsied and identified using immunohistochemistry, and representative images for the three germ layers were observed using an inverted microscope (80i, Nikon, Inc., Tokyo, Japan).

\section{Results}

Generation of heart disease-specific iPSCs using mouse feeder layer cells or feeder-free conditions. Six iPSC lines were derived successfully using fibroblast cells from patients with MI (Fig. 1A), including three cell lines using feeder-layer cells (HTH-iPS-1-3) and three cell lines generated under feeder-free conditions (HTH-iPS-4-6). Small colonies were able to be observed on day 12 after transfection with four transcription factors, and the primary colonies were passaged on day 20 (Fig. 1B). The cells had normal stem cell morphology and exhibited the features of human ESCs, including a high nucleus/cytoplasm ratio and tightly packed colonies when observed under an inverted microscope. There were no differences in the morphology of iPSCs grown under different culture conditions (Fig. 1C and D). The iPSCs showed high levels of alkaline phosphatase activity, and had normal karyotypes following long-term propagation (Fig. 2A-C). 

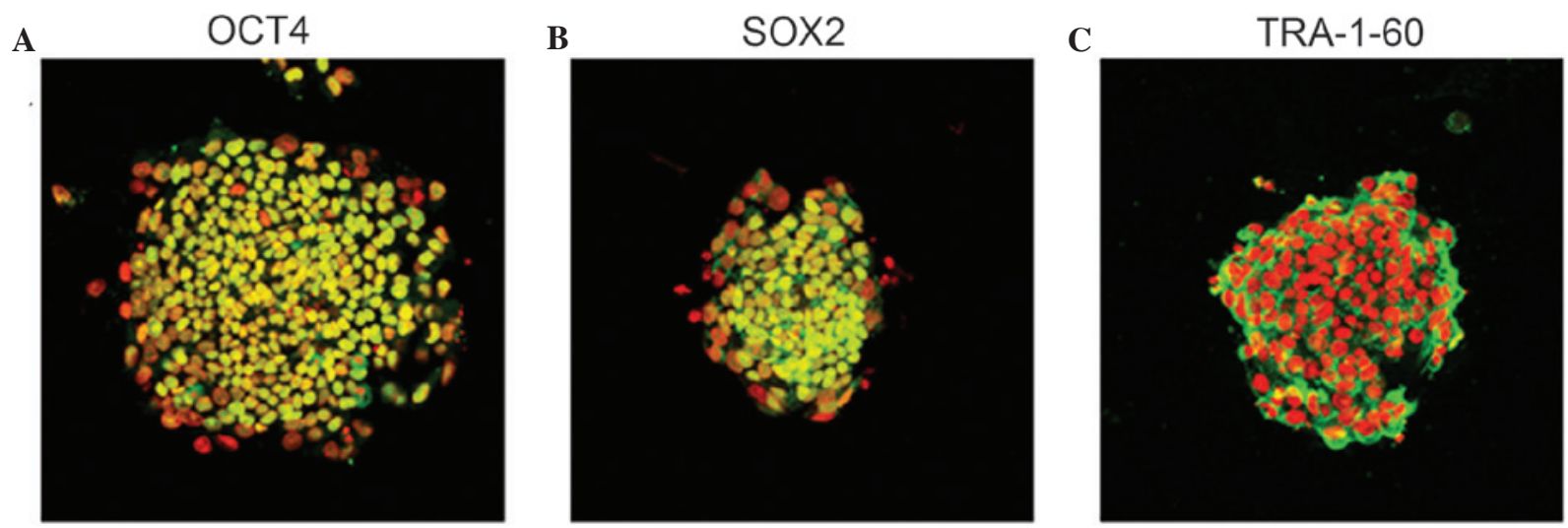

Figure 3. Visualization of pluripotent and cell surface markers in human iPS cells by immunofluorescence. (A) OCT-4 expression; (B) SOX-2 expression; (C) TRA-1-60 expression. iPS, induced pluripotent stem cells; Oct-4, octamer-binding transcription factor 4; Sox-2, SRY-related HMG-box gene 2; TRA-1-60, tumor rejection antigen-1-60.
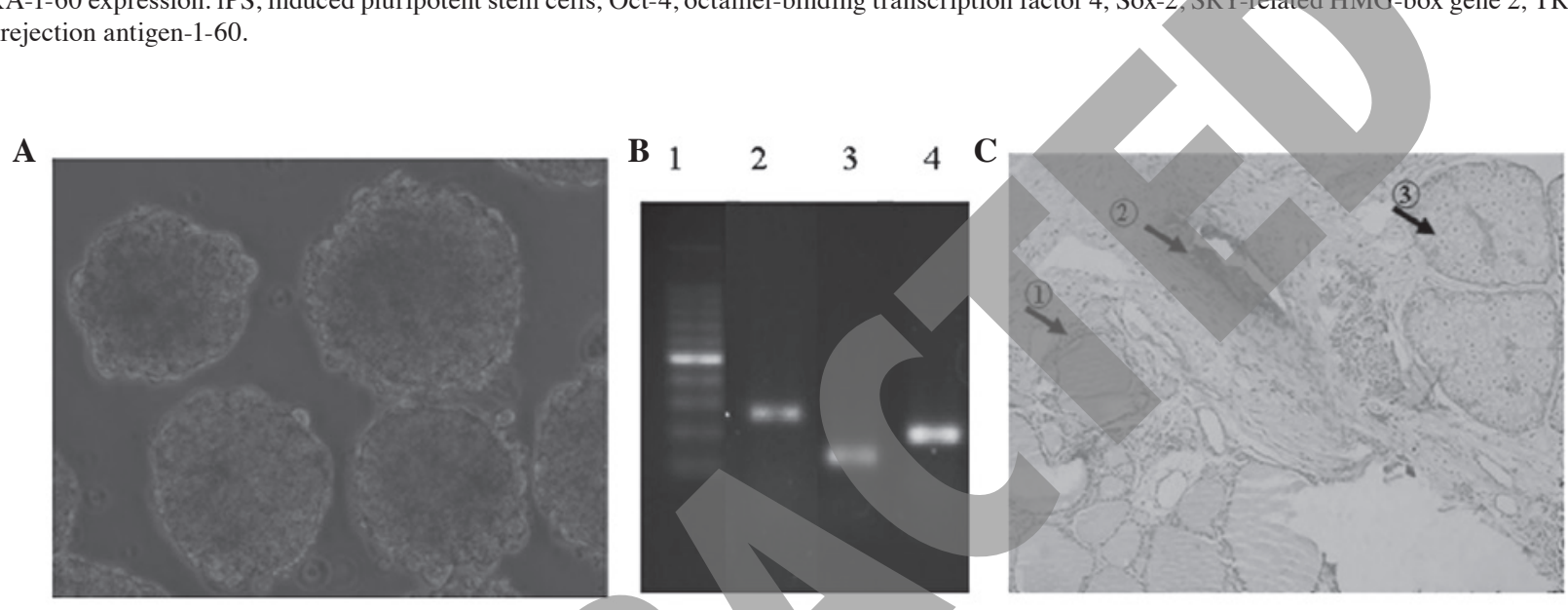

Figure 4. Identification of differentiation ability of human iPS cells in vitro and in vivo. (A)EB formation; (B) RT-PCR results for EB derivatives (lane 1, 100 bp markers; lane 2, GATA4, endoderm marker; lane 3, ACTA4, mesoderm marker; lane 4, NES, ectoderm marker); (C) teratoma formation and HE identification for the derivatives from three embryonic germ layers (arrow 1 indicates the thyroid that belongs to the endoderm, arrow 2 indicates the cartilage that belongs to the mesoderm and arrow 3 indicates the sebaceous gland that belongs to the ectoderm). iPS, induced pluripotent stem cells; RT-PCR, reverse transcription polymerase chain reaction; bp, base pairs; EB, embryoid body; GATA4, GATA binding protein 4; ACTA4, actin, alpha 4, smooth muscle, aorta; NES, nestin; HE, hematoxylin and eosin. Magnification, x100.

Characteristics of human heart disease-specific iPSCs. To identify the characteristics of human heart disease-specific iPSCs, cell surface markers were detected by immunofluorescence and pluripotent gene expression was detected by RT-PCR. All iPSCs expressed the pluripotent markers Oct-4 and Nanog, and the cell surface marker TRA-1-60 (Fig. 3A-C).

Differentiation ability of human heart disease-specific iPSCs. To evaluate the differentiation abilities of the iPSC lines, the ability of the iPSCs to differentiate into EBs in vitro when cultured in suspension was assessed. Following spontaneous differentiation for 7-10 days, the clumps of cells formed EBs, which were then subjected to RT-PCR analysis (Fig. 4A). Genes uniquely expressed in all three embryonic germ layers were detected, including AFP (endoderm), NEUOD-1 (ectoderm) and HBZ (mesoderm) (Fig. 4B).

Furthermore, the in vivo differentiation ability of the iPSCs was evaluated by teratoma formation. All the human iPSCs formed teratomas following injection into the back leg of SCID mice. Histopathological examination of the resulting teratomas revealed the presence of various tissue types, including thyroid (endoderm), cartilage (mesoderm) and seba- ceous gland (ectoderm) (Fig. 4C). These results demonstrate that human iPSCs are capable of differentiating into derivatives of all three embryonic germ layers in vivo.

\section{Discussion}

In the present study, human iPSCs were generated from skin fibroblast cells biopsied from patients with heart disease. To enhance the potential applications of these cells in clinical settings, feeder-free conditions were applied for iPSC generation and long-term propagation. All the generated iPSCs expressed pluripotent genes and specific cell surface markers, and had the ability to differentiate in vitro and in vivo to form derivatives of all three germ layers.

Pluripotent stem cells have shown tremendous potential in cell therapy, especially for the treatment of diseases with no other effective therapies. The successful derivation of mouse and human ESCs represents a promising strategy for the treatment of patients affected by diseases with no available cures; however, immune rejection currently limits their clinical application, as attempts to derive cloned human ESCs from patient somatic cells has consistently been unsuc- 
cessful $(22,23)$. Yamanaka and Takahashi (1) discovered that four transcription factors (Oct4, Sox2, Klf4 and c-Myc) were able to reverse the programming of terminally-differentiated mouse somatic cells back to pluripotent status (1), and the same group subsequently achieved similar results with human iPSCs (4). Hanna et al (24) proved that these cells had similar functions to human ESCs, and demonstrated the potential of iPSCs in cell therapy. In 2008, Park et al (7) were the first to derive human disease-specific iPSCs successfully, followed by success from other labs $(25,26)$.

Heart disease is, alongside cancer, regarded as one of the most lethal diseases. However, numerous heart diseases lack effective treatments. Heart disease-specific iPSCs have recently been derived from patients with Down syndrome, providing a valuable tool for exploring the pathogenesis of and developing novel treatment strategies for these diseases (9). The present study focused on MI, which is associated with the death of cardiomyocytes. Schächinger et al (27) indicated that bone marrow-derived progenitor cells may be able to improve the symptoms of acute MI following their injection into the coronary artery (27); however, no MI iPSCs have been reported to date. The present study therefore provides, to the best of our knowledge, the first report of the generation of MI-specific iPSCs, and their differentiation back to cardiomyocytes with typical morphology and characteristic cell marker expression.

Feeder cells have been regarded as an important factor for the derivation and growth of pluripotent stem cells. Fibroblast cells from 12.5-day-old mouse embryos have been extensively used for this purpose; however, their animal origin inhibits the development and clinical application of these stem cells. Human-homologous feeder cells have also been used to support the growth of human iPSCs, however feeder-free conditions have remained in the focus of research $\mathrm{Xu}$ et al (20) first indicated that human ESCs were able to maintain pluripotency under feeder-independent conditions, and Klimanskaya et al (19) successfully derived and cultured long-term human ESCs without feeder cells in 2005. Ludwig et al (28) established a simple serum-free, animal product-free medium to support the derivation and long-term feeder-independent culture of human ESCs, which was used in the present study. Sun et al (21) generated human iPSCs from adult human adipose stem cells, and Beltrão-Braga et al (29) subsequently derived iPSCs from human immature dental pulp stem cells without feeder cells. Recently, Macarthur et al (30) suggested that human neonatal fibroblast cells were able to be reprogrammed by transcription factors using a xeno-free culture system.

In conclusion, the present study generated MI-iPSCs that were able to be derived and propagated under feeder-independent conditions. These cells may be valuable for the study of MI pathogenesis, and are likely to facilitate the safe downstream clinical applications of iPSC-based cell therapies.

\section{Acknowledgements}

This study was supported by the Technological Innovation Talent Special Foundation of Harbin, China (2009RFXXS018) and the Youth Science Foundation of Heilongjiang Province (QC2013C116).

\section{References}

1. Takahashi K, Yamanaka S: Induction of pluripotent stem cells from mouse embryonic and adult fibroblast cultures by defined factors. Cell 126: 663-676, 2006.

2. Liao J, Cui C, Chen S, et al: Generation of induced pluripotent stem cell lines from adult rat cells. Cell Stem Cell 4: 11-15, 2009.

3. Liu H, Zhu F, Yong J, et al: Generation of induced pluripotent stem cells from adult rhesus monkey fibroblasts. Cell Stem Cell 3: 587-590, 2008

4. Takahashi K, Tanabe K, Ohnuki M, et al: Induction of pluripotent stem cells from adult human fibroblasts by defined factors. Cell 131: 861-872, 2007.

5. Maehr R, Chen S, Snitow M, et al: Generation of pluripotent stem cells from patients with type 1 diabetes. Proc Natl Acad Sci USA 106: 15768-15773, 2009.

6. Thatava T, Armstrong AS, De Lamo JG, et al: Successful disease-specific induced pluripotent stem cell generation from patients with kidney transplantation. Stem Cell Res Ther 2: 48, 2011.

7. Park IH, Arora N, Huo H, et al: Disease-specific induced pluripotent stem cells. Cell 134: 877-886, 2008.

8. Li LB, Chang KH, Wang PR, et al: Trisomy correction in Down syndrome induced pluripotent stem cells. Cell Stem Cell 11: 615-619, 2012.

9. Mou X, Wu Y, Cao H, et al: Generation of disease-specific induced pluripotent stem cells from patients with different karyotypes of Down syndrome. Stem Cell Res Ther 3: 14, 2012.

10. Valensi P, Lorgis L and Cottin Y: Prevalence, incidence, predictive factors and prognosis of silent myocardial infarction: a review of the literature. Arch Cardiovasc Dis 104: 178-188, 2011. Boheler KR, Czyz J, Tweedie D, et al: Differentiation of pluripotent embryonic stem cells into cardiomyocytes. Circ Res 91: 189-201, 2002.

12. Passier R, Oostwaard DW, Snapper J, et al: Increased cardiomyocyte differentiation from human embryonic stem cells in serum-free cultures. Stem Cells 23: 772-780, 2005.

3. Lian X, Hsiao C, Wilson G, et al: Robust cardiomyocyte differentiation from human pluripotent stem cells via temporal modulation of canonical Wnt signaling. Proc Natl Acad Sci USA 109: E1848-E1857, 2012.

4. Zwi L, Caspi O, Arbel G, et al: Cardiomyocyte differentiation of human induced pluripotent stem cells. Circulation 120: 1513-1523, 2009

15. Thomson JA, Itskovitz-Eldor J, Shapiro SS, et al: Embryonic stem cell lines derived from human blastocysts. Science 282: 1145-1147, 1998.

16. Martin MJ, Muotri A, Gage F and Varki A: Human embryonic stem cells express an immunogenic nonhuman sialic acid. Nat Med 11: 228-232, 2005.

17. Lu Z, Zhu W, Yu Y, et al: Derivation and long-term culture of human parthenogenetic embryonic stem cells using human foreskin feeders. J Assist Reprod Genet 27: 285-291, 2010.

18. Zhang YS, Lu ZY, Yu Y, et al: Derivation, culture and retinal pigment epithelial differentiation of human embryonic stem cells using human fibroblast feeder cells. J Assist Reprod Genet 29: 735-744, 2012.

19. Klimanskaya I, Chung Y, Meisner L, et al: Human embryonic stem cells derived without feeder cells. Lancet 365: 1636-1641, 2005.

20. Xu C, Inokuma MS, Denham J, et al: Feeder-free growth of undifferentiated human embryonic stem cells. Nat Biotechnol 19: 971-974, 2001.

21. Sun N, Panetta NJ, Gupta DM, et al: Feeder-free derivation of induced pluripotent stem cells from adult human adipose stem cells. Proc Natl Acad Sci USA 106: 15720-15725, 2009.

22. French AJ, Adams CA, Anderson LS, et al: Development of human cloned blastocysts following somatic cell nuclear transfer with adult fibroblasts. Stem Cells 26: 485-493, 2008.

23. Yu Y, Mai Q, Chen X, et al: Assessment of the developmental competence of human somatic cell nuclear transfer embryos by oocyte morphology classification. Hum Reprod 24: 649-657, 2009.

24. Hanna J, Wernig M, Markoulaki S, et al: Treatment of sickle cell anemia mouse model with iPS cells generated from autologous skin. Science 318: 1920-1923, 2007.

25. Chun YS, Chaudhari P and Jang YY: Applications of patient-specific induced pluripotent stem cells; focused on disease modeling, drug screening and therapeutic potentials for liver disease. Int J Biol Sci 6: 796-805, 2010. 
26. Ye Z, Zhan H, Mali P, et al: Human-induced pluripotent stem cells from blood cells of healthy donors and patients with acquired blood disorders. Blood 114: 5473-5480, 2009.

27. Schächinger V, Erbs S, Elsässer A, et al; REPAIR-AMI Investigators: Intracoronary bone marrow-derived progenitor cells in acute myocardial infarction. N Engl J Med 355: 1210-1221, 2006

28. Ludwig TE, Bergendahl V, Levenstein ME, et al: Feeder-independent culture of human embryonic stem cells. Nat Methods 3: 637-646, 2006
29. Beltrão-Braga PI, Pignatari GC, Maiorka PC, et al: Feeder-free derivation of induced pluripotent stem cells from human immature dental pulp stem cells. Cell Transplant 20: 1707-1719, 2011.

30. Macarthur CC, Fontes A, Ravinder N, et al: Generation of human-induced pluripotent stem cells by a nonintegrating RNA Sendai virus vector in feeder-free or xeno-free conditions. Stem Cells Int 2012: 564612, 2012. 